\title{
Factores clave de éxito en proyectos de software en el sector financiero colombiano
}

Fecha de recepción: 4 de septiembre de 2017

Fecha de aprobación: 24 de diciembre de 2017

DOI: https://doi.org/10.21158/23823399.v5.n0.2017.1992
Jeisson Alfonso Córdoba Zambrano

Universidad EAN

jeisson.cordoba@gmail.com

John Edwin Garcia Ospina

Universidad EAN

jx8213@gmail.com

Yuly Milena Ramírez Guilombo

Universidad EAN

yumilena87@gmail.com

Cómo citar este artículo/To reference this article/Comment citer cet article/Para citar este artigo: Córdoba Zambrano, J. A.; García Ospina, J. E. y Ramírez Guilombo, Y. M. (2017). Factores clave de éxito en proyectos de software en el sector financiero colombiano. Revista Ontare. 5, p 7-48.

\section{Resumen}

En la presente investigación se explicarán las propiedades y características de los proyectos de software en Colombia, realizando análisis y mediciones para identificar los factores de éxito y fracaso en los proyectos de software del sector financiero colombiano con duración de más de dos años y con un presupuesto mayor a 2 millones de dólares. En la primera sección se presentan la justificación y objetivos de la investigación, enseguida se plantea la problemática a tratar, el marco de referencia, la hipótesis, la propuesta y por ultimo las conclusiones.

\section{Palabras clave}

Factores de éxito; proyectos de software; software financiero; sistema financiero colombiano; tecnologías de la información.

1 Ingeniero de sistemas de la fundación universitaria San Martin, con especialización en gerencia informática de la universidad EAN, Scrum master del scrum agile institute, Project Manager Devops de Devops Academy.

2 Ingeniero de Sistemas de la Universidad de Cundinamarca, Especialista en Gerencia Informática de la Universidad EAN y Diplomado en Gerencia de Proyectos de la Universidad de Cundinamarca.

3 Ingeniera de Sistemas - Universidad Autónoma de Colombia. Tester Certificada ISTQB. Diplomado en Gestión de Tecnologías de la Información- Universidad Austral de Buenos Aires. Especialista en Gerencia Informática - Universidad EAN 


\title{
Key success factors in software projects in the Colombian financial sector
}

\begin{abstract}
The aim of this investigation is to explain the properties and characteristics of the software projects in Colombia, by making analyses and measurements in order to identify the success and failure factors in the software projects of the Colombian financial sector with duration of more than two years, and a budget greater than 2 million dollars. In the first section, we present the justification and objectives of the research, then, we state the problem to be treated, the reference framework, the hypothesis, the proposal, and finally the conclusions.
\end{abstract}

\section{Keywords}

Success factors; software projects; financial software; Colombian financial system; information technologies.

\section{Facteurs clés de succès des projets logiciels du secteur financier colombien}

\section{Résumé}

Lors de cette investigation nous tenterons d'expliquer les propriétés et caractéristiques des projets logiciels en Colombie en analysant, mesurant et identifiant les facteurs de réussite et d'échec des projets logiciels du secteur financier colombien s'échelonnant sur une durée de plus de deux ans et dotés d'un budget d'au moins 2 millions de dollars. Nous produirons premièrement une justification de notre investigation et les objectifs de notre recherche avant d'aborder dans une seconde partie la problématique à traiter, le cadre de référence, l'hypothèse et la proposition avant de conclure ce travail.

\section{Mots clefs}

Facteurs de succès; projets logiciels; logiciels financiers; système financier colombien; technologies de l'information. 


\section{Fatores chave de sucesso em projetos de software no setor financeiro colombiano}

\section{Resumo}

Na presente pesquisa explicar-se-ão as propriedades e características dos projetos de software na Colômbia, realizando análise e medições para identificar os fatores de sucesso e fracasso nos projetos de software do setor financeiro colombiano com duração de mais de dois anos e com um orçamento maior a 2 milhões de dólares. $\mathrm{Na}$ primeira seção apresentam-se a justificativa e os objetivos da pesquisa, em seguida propõe-se a problemática a tratar, o marco de referência, a hipótese, a proposta e por último as conclusões.

\section{Palavras-chave}

Fatores de sucesso; projetos de software; software financeiro; sistema financeiro colombiano; tecnologias da informação. 
Tn la actualidad, el dinamismo, el tamaño y la duración, entre otros 1 factores de los proyectos de software, generan una serie de variables por tener en cuenta y que deben ser controladas para finalizar el proyecto de manera satisfactoria. Sin embargo, a pesar de tener conocimiento de ello, en muchas ocasiones, no se tienen en cuenta todos los elementos o no se tratan a fondo y esto causa problemas en el proyecto, los que pueden impactar el tiempo, los costos, el alcance y la calidad comprometida, y llevarlo al fracaso.

\section{Justificación}

11 presente artículo describirá los factores clave de éxito que se deben Ctener en cuenta en la planeación y el desarrollo de los proyectos de software en el sector financiero y cuáles son los factores de fracaso que han afectado a los proyectos de software en este sector.

Se pretende elaborar un artículo con enfoque gerencial, que ayude al lector a entender la importancia de una buena planeación en proyectos de software financiero, y de este este modo mostrar un análisis de los factores mencionados para fortalecer la toma de decisiones a la hora de planear y hacer énfasis en su importancia para el desarrollo y la culminación de proyectos que cumplan con las metas de presupuesto, tiempos, alcance y calidad.

En el ámbito del software financiero, es de vital importancia que los proyectos salgan bien, no solo basta con que cumplan con los requerimientos establecidos, sino que presenten un alto nivel de calidad, ya que vivimos en un mundo capitalista, donde todo gira alrededor de la economía y las finanzas, por lo que las fallas cometidas en estas áreas pueden tener graves consecuencias para un país (King, 2014). 
El sector financiero requiere que su software cumpla con los estándares de calidad más exigentes del mercado. Esto incluye altas tasas de eficiencia en rendimiento, usabilidad, estabilidad, disponibilidad y confiabilidad, tal y como se describe en el actual estándar de calidad de software ${ }^{1}$ ISO 25000 (Iso25000.com, s. f.). Sin embargo, existen casos muy graves de falencias producidas en el sector, como el reciente de un «mínimo» error de software que causó una fluctuación en el índice bursátil Dow Jones (King, 2014). Este tipo de errores son inaceptables, ya que, como el caso en mención, pueden generar consecuencias graves, y como si se trataran de una bola de nieve, afectan gravemente la economía de las empresas y su sostenibilidad en un momento dado.

El desarrollo del software financiero es cada vez más complejo, debido a su interoperabilidad con múltiples sistemas de información, inter-bancarios, internacionales, interrelacionados y unificados con software de terceros, y aparte de todo esto, deben convivir con aplicaciones heredadas creadas por diferentes equipos de desarrollo y que aún continúan en funcionamiento por su complejidad y eficiencia (Partnov y Estier, 2009). Estos puntos hacen que el software financiero sea cada vez más complejo y, por consiguiente, que su mantenibilidad sea un duro trabajo donde cualquier error derivará en contra de los usuarios del servicio financiero directamente (Kelion, 2013). Estos factores deben ser analizados, docu-mentados y considerados para la realización y culminación exitosa de proyectos de software financiero.

1 ISO 25000: el objetivo general de la creación del estándar es organizar, enriquecer y unificar las series que cubren dos procesos principales: especificación de requisitos de calidad del software y evaluación de su calidad. 


\section{Objetivo general}

- Determinar los factores clave de éxito en los proyectos de software del sector financiero colombiano con duración de más de dos años y con un presupuesto mayor de USD 2 millones.

\section{Objetivos específicos}

- Identificar los factores clave del éxito de proyectos de software.

- Identificar los factores que llevaron al fracaso a los proyectos de software.

- Seleccionar y analizar los factores de éxito por tener en cuenta en las fases de iniciación, planeación, ejecución, control y cierre.

- Seleccionar y analizar los factores de fracaso por tener en cuenta para analizar y controlar en mayor detalle en las fases de iniciación, planeación, ejecución, control y cierre.

\section{Tipo de investigación}

T a investigación realizada es descriptiva, en la que se explican las Upropiedades y características de los proyectos de software en Colombia, realizando análisis y mediciones para identificar los factores de éxito y fracaso (Cortés e Iglesias, 2005).

\section{Problemática}

e ha detectado que la mayoría de los proyectos de software fracasan porque no están bien planeados, existen factores que no se tienen en cuenta o que se obvian y que solo en el desarrollo del proyecto se 
evidencia que hicieron falta en la planeación. También se presenta el caso de algunos factores que, a pesar de tenerlos en cuenta, no se analizaron o no se enfocaron de la forma debida para el proyecto. McKinsey \& Company y la Universidad de Oxford en 2012 llevaron a cabo un estudio enfocado en proyectos grandes de tecnología de la información (TI), cuyo presupuesto inicial era mayor de USD 15 millones, tras lo cual la investigación encontró que en estos proyectos el $45 \%$ excedieron el presupuesto, el $7 \%$ sobrepasaron el cronograma y el $56 \%$ entregaron menos de lo comprometido (Bloch, Blumberg y Laartz, 2012).

La figura 1 muestra el desempeño de diferentes proyectos de tecnología en los tres factores mencionados - alcance, tiempo y costo-.

Figura 1. Desempeño de diferentes tipos de proyectos de TI

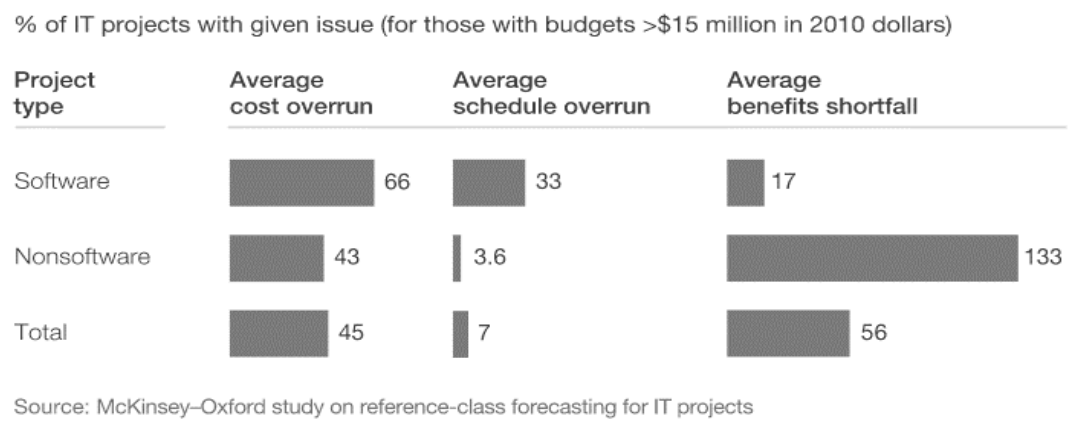

Fuente. McKinsey \& Company, 2014.

La mayoría de las empresas, aunque tambaleando, logran sobrevivir a los altos costos y retrasos que evidencia el estudio. Sin embargo, el $17 \%$ de los proyectos pueden tener un resultado tan negativo que ponen en riesgo a la empresa (Evans y Ganegoda, 2012). Los eventos inesperados de alto impacto se conocen como cisnes negros. Grandes proyectos de TI que se convierten en cisnes negros se definen como aquellos que exceden el presupuesto en más del $200 \%$. Existen tres categorías de cisnes negros: 
- Conocido/conocido: se sabe que el riesgo existe y se sabe cómo se comportará y cuál será su impacto.

- Conocido/desconocido: se sabe que el riesgo existe, pero no se tiene confiabilidad de cómo o cuándo ocurrirá y qué impacto generará.

- Desconocido/desconocido: no se tiene idea de que existirá el riesgo.

Además, muchas de las fallas cometidas durante el proceso de desarrollo de un producto software son provocadas por el bajo interés en seguir la metodología establecida para hacer una adecuada planeación (Dorsey, 2005), muchas veces influenciado por el afán de «producir», de ver resultados pronto, de este modo descuidando aspectos fundamentales de una adecuada planeación que se verán reflejados en la calidad final del producto y harán, incluso, que se obtengan funcionalidades que no son útiles para el usuario, es decir que no cumplen con los requerimientos funcionales que el cliente desea en su producto.

Según Lyytinen y Hirschheim (1988), se pueden definir cuatro tipos de problemas en los proyectos de software que fracasan: el primero ocurre cuando el diseño del sistema no cumple con los objetivos especificados por el usuario; el segundo cuando un proyecto excede el presupuesto y tiempo asignado en la planeación; el tercero cuando el sistema desarrollado no supera los niveles de utilización esperados y la percepción del usuario es que lo usa porque no hay otra opción y el cuarto cuando el sistema no supera las expectativas del usuario en cuanto al funcionamiento. Se debe encontrar una solución a cada una de estos problemas para garantizar mayor éxito en los proyectos de software. Otros aspectos que llevan al fracaso de los proyectos y que se deben revisar a fondo son la falta de participación del usuario en el desarrollo del proyecto, ya que se debe involucrar al usuario para que ayude a aclarar los requerimientos y así desarrollar realmente lo que él espera y no lo que el desarrollador cree que este espera, y que a los requerimientos no funcionales y de calidad se les debe dar la misma importancia que a los requerimientos funcionales, 
puesto que se tiende a enfocar el sistema en cumplir los requerimientos funcionales, pero no se visualiza que los requerimientos no funcionales y de calidad están embebidos en lo que el usuario necesita así no lo manifieste explícitamente.

Reel (1999) señala diez síntomas que presentan los proyectos que probablemente fracasarán:

- Los gerentes del proyecto no entienden las necesidades de los usuarios.

- No está bien definido el alcance del proyecto.

- No existe un proceso formal de control de cambios y por ende estos se gestionan mal.

- Se realizan cambios en las tecnologías inicialmente definidas.

- Se presentan cambios en el negocio.

- Definición de tiempos y cronogramas no realistas.

- Usuarios resistentes.

- Pérdida del respaldo del patrocinador del proyecto.

- El proyecto no cuenta con el equipo de trabajo que tenga las habilidades necesarias para este.

- El gerente ignora las buenas prácticas y lecciones aprendidas.

Con una serie de entrevistas realizadas por McKinsey \& Company en 2010 a un grupo de ejecutivos de TI, se identificaron cuatro dimensiones que impulsan el éxito de los proyectos al tenerlas combinadas y correctamente administradas (Figura 2):

- Centrar toda la atención en la gestión de los interesados y en la estrategia más que en el presupuesto y el cronograma.

- Dominio de la tecnología utilizada en el proyecto, de modo que asegure recursos tanto internos como externos.

- Creación de equipos eficaces, con motivaciones alineadas a los objetivos del proyecto.

- Buenas prácticas de gestión de proyectos como ciclos de entrega cortos y rigurosos controles de calidad. 
Las encuestas arrojaron que la falta de capacidad para gestionar las dos primeras dimensiones en general causaba la mitad de todos los sobrecostos, mientras que los malos resultados en la gestión de las últimas dos dimensiones representan un $40 \%$ más de gastos.

Figura 2. Cuatro dimensiones que causan la mayoría de los fracasos en proyectos

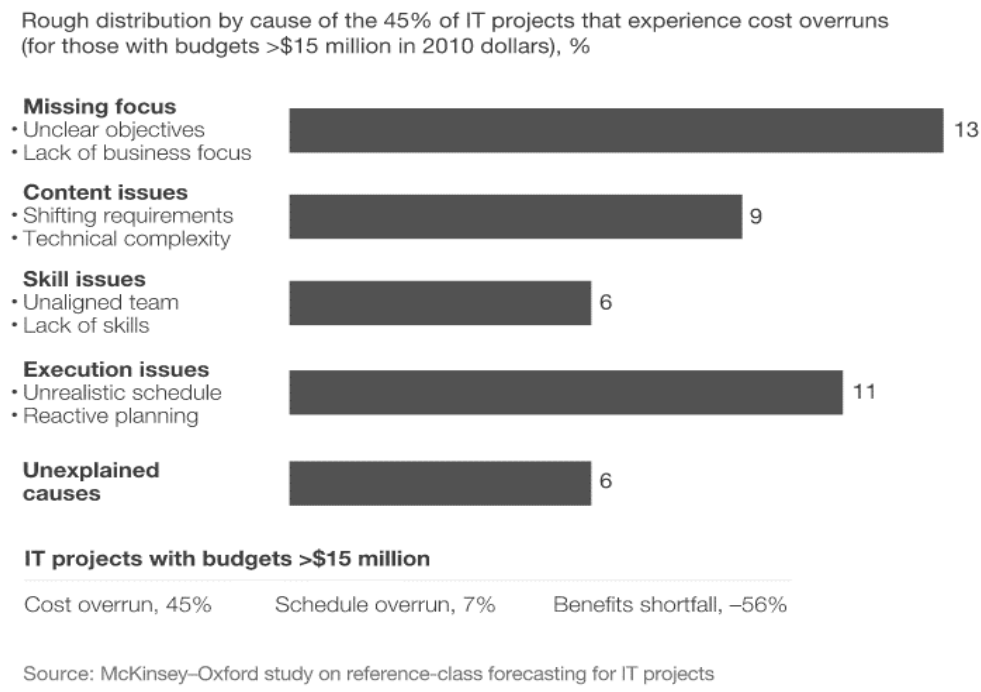

Fuente. McKinsey \& Company, 2014.

En Colombia, se han hecho algunos estudios y reflexiones en cuanto a los errores que se cometen y factores de fracaso en los proyectos de software, muchos de estos se viven a diario en gran parte de las empresas colombianas. Ocampo (2013) señala algunos:

- Falta de sensibilidad y conocimiento del negocio al que está contribuyendo el proyecto de software: de lo anterior se deduce que el conocimiento en el negocio es vital para que el proyecto aporte en realidad al cliente, pero, por lo general, el mayor esfuerzo se da en crear el producto sin ver si verdaderamente cumple con las reglas de negocio y aporta valor a los procesos que se llevan a cabo en la empresa. 
- Nos enfocamos en programar la funcionalidad, pero la información no es relevante para nosotros: se puede inferir que en nuestro país nos enfocamos siempre en crear la parte funcional, pero los requerimientos no son examinados en detalle para elaborar las pruebas funcionales basándose en esta información para estar alineados con los objetivos del cliente.

- La falta de ejecución sistemática de la disciplina de administración de la configuración genera quiebres entre la operación y el desarrollo: muchas veces, el proceso de desarrollo del proyecto puede no estar apoyado en una adecuada gestión de la configuración que proporcione un manejo pertinente de ambientes, sistemas de versionamiento, configuración de herramientas de prueba, auditoría de estas configuraciones, etc.

- Falta disciplina en la administración de requerimientos y en la administración de pruebas: las pruebas muchas veces se pueden llegar a subvalorar, pero se deberían aplicar como una parte integral del software debido a que evalúan su calidad y a la forma en la que el software va a responder a las necesidades reales del cliente.

- A pesar de que se configuran equipos de trabajo interdisciplinarios para algunos proyectos, se subestiman el impacto de las interacciones, las culturas y los procesos de dichos equipos: por lo general, se pasan por alto las relaciones entre los equipos de trabajo involucrados en el transcurso del proyecto. No se tiene en cuenta si estos tienen diferentes formas de trabajar y distintas culturas empresariales que al interactuar pueden generar roces entre los miembros del equipo o provocar inconvenientes que pueden desembocar en la poca eficiencia de los integrantes del proyecto y en los procesos que estos llevan a cabo.

- Existe una tendencia de aplicar las metodologías tradicionales en los proyectos de software - y un temor de aplicar metodologías ágiles-: según esto, las metodologías tradicionales reinan en Colombia. Su implementación puede llegar a ser muy rigurosa y no ser la respuesta a las necesidades del país. Esto puede derivar, incluso, en la no adopción de metodologías por parte 
de ciertas empresas que pueden preferir crear las propias, y relegar, incluso, la implementación de frameworks que han ayudarles como los propuestos por las metodologías ágiles.

Salinas (2007), basándose en una investigación del grupo Standish, señala algunos de los factores de fracaso en los proyectos de TI genéricos que pueden ser aplicables a nuestro país:

- requerimientos incompletos

- no involucrar al usuario

- expectativas no realistas

- cambios en los requerimientos y especificaciones

- deficiencia en la planeación

- deficiencia en la administración de TI

- desconocimiento en tecnología

El estudio realizado por Wiklund y Pucciarelli (2009) indica que el $20 \%$ de los proyectos fracasan súbitamente, entre el 20 y el $25 \%$ no proporcionan el retorno de la inversión y el $50 \%$ requieren retrabajo. Los problemas se reducen a siete razones principales, que se describen a continuación:

- planificación y dirección de proyecto pobre

- comunicación insuficiente

- gestión ineficaz

- no alineación entre la dirección del proyecto y los interesados

- participación ineficaz de la alta gerencia

- falta de habilidades sociales y capacidad de adaptación

- deficiencia o falta de metodología y herramientas 
En el estudio realizado por McManus y Wood-Harper (2007), se analizaron 214 proyectos de TI en Europa entre 1998 y 2005, en los sectores que muestra la figura 3 .

Figura 3. Número de Proyectos de TI analizados en la Comunidad Europea

\begin{tabular}{|c|l|c|}
\hline Rank & Sector & Number of Projects Examined \\
\hline 1 & Manufacturing & 43 \\
\hline 2 & Retail & 36 \\
\hline 3 & Financial Services & 33 \\
\hline 4 & Transport & 27 \\
\hline 5 & Health & 18 \\
\hline 6 & Education & 17 \\
\hline 7 & Defence & 13 \\
\hline 8 & Construction & 12 \\
\hline 9 & Logistics & 9 \\
\hline 10 & Agriculture Total & 6 \\
\hline \multicolumn{2}{r|}{} & \\
\cline { 2 - 3 } & & 214 \\
\hline
\end{tabular}

Fuente McManus y Wood-Harper, 2007.

El estudio encontró que el $23,8 \%$ de los proyectos -51 proyectos, casi la cuarta parte- fueron cancelados después de la etapa de factibilidad. La que cancelación de estos proyectos se dio por la combinación de factores como (Figura 4):

- alineación de los procesos de negocio

- pobre gestión de requerimientos

- beneficios exagerados

- diferencias entre la gerencia y el cliente

- falta de criterio de la gerencia

- conocimiento pobre o insuficiente

- pérdida de personal clave

- pobre comunicación con los interesados 
- sistemas de información pobres;

- ausencia de un proceso de control de cambios.

Figura 4. Razones principales por las que fueron cancelados los 51 proyectos

\begin{tabular}{|c|c|c|}
\hline Business reasons $(N=10)$ & Management reasons $(N=27)$ & Technical reasons $(\mathrm{N}=14)$ \\
\hline $19.6 \%$ & 53.08 & 27.45 \\
\hline - Business strategy superseded & - Ability to adapt to new resource combinations & - Inappropriate architecture \\
\hline - Business processes change (poor alignment) & - Differences between management and client & - Insufficient reuse of existing technical objects \\
\hline - poor requirements management & - Insufficient risk management & - Inappropriate testing tools \\
\hline $\begin{array}{l}\text { - Business benefits not clearly communicated } \\
\text { or overstated }\end{array}$ & - Insufficient end-user management & - Inappropriate coding language \\
\hline - Failure of parent company to deliver & - Insufficient domain knowledge & - Inappropriate technical methodologies \\
\hline - Governance issues within the contract & - Insufficient software metrics & - Lack of formal technical standards \\
\hline - Higher cost of capital & - Insufficient training of users & - Lack of technical innowation (obsolescence) \\
\hline - Inability to provide investment capital & - Inappropriate procedures and routines & - Misstatement of technical risk \\
\hline - Inappropriate disaster recovery & - Lack of management judgement & - Obsolescence of technology \\
\hline - Misuse of financial resources & - Lack of software development metrics & - Poor interface specifications \\
\hline - Overspends in excess of agreed budgets & - Loss of key personnel & - Poor quality code \\
\hline - Poor project board composition & - Managing legacy replacement & - Poor systems testing \\
\hline - Take-over of client firm & - Poor vendor management & - Poor data migration \\
\hline \multirow[t]{7}{*}{\begin{tabular}{|l} 
- Too big a project portfolio \\
\end{tabular}} & - Poor software productivity & - Poor systems integration \\
\hline & - Poor communication between stakeholders & - Poor configuration management \\
\hline & - Poor contract management & - Poor change management procedures \\
\hline & - Poor financial management & - Poor technical judgement \\
\hline & - Project management capability & \\
\hline & - Poor delegation and decision making & \\
\hline & - Unfilled promises to users and other stakeholde & \\
\hline
\end{tabular}

Fuente. McManus y Wood-Harper, 2007

McManus y Wood-Harper (2007) encontraron que los proyectos que duran más de 26 meses y tienen un presupuesto superior a los USD 6 millones incurrían en excesos de costos o cronograma. En estos casos, el gerente de proyecto debía actuar; sin embargo, la búsqueda de factores debe realizarse en las lecciones aprendidas e históricos de los proyectos, pero encontrar y sintetizar esta información puede ser muy complejo y ambiguo, además los puntos de vista de los interesados para dar solución a los sobrecostos o desviación del cronograma pueden ser muy diferentes de los que plantea el gerente del proyecto. 
El estudio indica que de los proyectos terminados aproximadamente uno de cada tres estaba excedido en presupuesto o cronograma. La figura 5 muestra un resumen de los proyectos con desviación en costo o cronograma.

Figura 5. Proyectos con exceso en costo o cronograma

\begin{tabular}{|l|c|c|c|c|c|}
\hline $\begin{array}{l}\text { Projects } \\
\text { From } \\
\text { Sample }\end{array}$ & $2(2)$ & $11(13)$ & $19(32)$ & $25(57)$ & $12(69)$ \\
\hline $\begin{array}{l}\text { Schedule } \\
\text { Overrun }\end{array}$ & 11 weeks & 29 weeks & 46 weeks & 80 weeks & 103 weeks \\
\hline Range & $\begin{array}{c}\text { Average } \\
\text { Budget }+ \\
10 \%\end{array}$ & $\begin{array}{c}\text { Average } \\
\text { Budget }+ \\
25 \%\end{array}$ & $\begin{array}{c}\text { Average } \\
\text { Budget }+ \\
40 \%\end{array}$ & $\begin{array}{c}\text { Average } \\
\text { Budget }+ \\
70 \%\end{array}$ & $\begin{array}{c}\text { Average } \\
\text { Budget }+ \\
90 \%\end{array}$ \\
\hline $\begin{array}{l}\text { Cost } \\
\text { Overrun }\end{array}$ & $\begin{array}{c}600,000 \\
\text { Euros }\end{array}$ & $\begin{array}{c}1,500,000 \\
\text { Euros }\end{array}$ & $\begin{array}{c}2,400,000 \\
\text { Euros }\end{array}$ & $\begin{array}{c}4,200,000 \\
\text { Euros }\end{array}$ & $\begin{array}{c}5,400,000 \\
\text { Euros }\end{array}$ \\
\hline
\end{tabular}

Fuente. McManus y Wood-Harper, 2007

Todo lo anterior muestra las diferentes características y síntomas que presentan los proyectos de tecnología, en diferentes sectores de la industria, donde se logran evidenciar factores comunes, y en algunos casos, factores que generan un mayor impacto y que aceleran el fracaso del proyecto.

\section{Marco de referencia}

«Un proyecto es un esfuerzo temporal que se lleva a cabo para crear un bien o servicio único» (Project Management Institute, 2013, p. 3). La característica de temporal indica que tiene un comienzo y un fin definido; el comienzo se da una vez el proyecto ha sido aprobado y su fin se presenta cuando se alcanza el objetivo para el que se creó el proyecto, cuando la necesidad que dio inicio al proyecto ya no existe o cuando el objetivo del proyecto no se cumplirá. En cuanto al bien, se refiere 
a un producto tangible y cuantificable creado por el proyecto; si es un servicio, se refiere a un trabajo útil que es intangible y tiene la capacidad de realizar una función o generar nuevo conocimiento. Por último, la característica de único indica que no hay dos entregables iguales, ya que un proyecto siempre tendrá distintos elementos como personas, ubicación o materiales; el entregable puede ser único en su género.

Domínguez (2007) indica que «un proyecto consiste en colocar/ utilizar los recursos para lograr un objetivo específico siguiendo un esquema planificado y organizado».

El proyecto también se define como «una secuencia bien definida de eventos con un principio y un final, dirigidos a alcanzar un objetivo claro y realizado por personas dentro de unos parámetros establecidos, tales como tiempo, costo, recursos y calidad» (Cano, 2006).

Project Management Institute (2013. pp. 5-6) indica que «la dirección de proyectos es la aplicación de conocimientos, habilidades, técnicas y herramientas a las actividades del proyecto para cumplir con los requerimientos mínimos». Y dentro de esas habilidades el gerente del proyecto debe «equilibrar las restricciones contrapuestas del proyecto», con lo cual se entiende que existe una relación muy estrecha entre los factores que afectan al proyecto de tal forma que si uno de estos cambia es probable que al menos otro se vea afectado. Los factores sobre los que se debe tener especial cuidado son el alcance, el tiempo y el costo, los que se entienden como «la triple restricción».

Un factor es un elemento que influencia positiva o negativamente un resultado, el éxito es el resultado feliz de un negocio o actuación y el fracaso es el resultado adverso de una empresa o negocio (Real Academia Española, 2014). De acuerdo con esto, se puede señalar que hay elementos que afectan de manera positiva y llevan a un resultado exitoso de alguna acción y hay factores que afectan de manera negativa y llevan a un resultado de fracaso en la acción que se emprendió. 
Este tipo de factores se pueden evidenciar en diferentes entornos como el personal, social, empresarial, cultural, entre otros. A continuación, se referencian diferentes definiciones de factores clave de éxito que han sido generadas a causa de investigaciones en diferentes entornos, las cuales se listan a continuación:

- «Los factores críticos de éxito son puntos de apoyo para el apalancamiento de una organización hacia el logro de resultados exitosos. Son dinámicos en tanto dependen de la coyuntura de cada negocio y debe desdoblarse a distintos niveles de recursividad de manera armónica a fin de garantizar el equilibrio entre las exigencias internas y externas al sistema» (Villegas, 1997, p. 4).

- «Podemos definir los factores claves de éxito como: el conjunto de condiciones y actividades del sistema, suficientes y necesarias para asegurar el logro de los objetivos del sistema, y que por lo tanto deben estar bajo control» (Bahamón, 2003, p. 82).

- «Los factores críticos de éxito son variables que se deben tener en cuenta antes y durante la realización de un proyecto, ya que aportan información valiosa para alcanzar las metas y objetivos de la empresa» (Romero, Noriega, Escobar y Ávila, 2009, p. 7).

- «Los factores críticos de éxito son aquellos factores que son críticos para el éxito de una organización, en el sentido que, si los objetivos que están asociados con el factor no se cumplen, entonces la organización fallará en el logro de sus metas y tal vez catastróficamente» (Rockart, 1979).

De acuerdo con estas nociones, se pueden definir los factores clave de éxito como un conjunto de acciones que se deben identificar y seguir para el logro de los objetivos propuestos. Por tanto, el hecho de no darles la importancia requerida a estos pueden convertirlos en factores de fracaso e impedir el logro del objetivo planteado. 
Los fracasos en los proyectos financieros están ligados a la falta de visión, de planeación en el futuro, de ver los diferentes objetivos y características que ha de cubrir el software y el medio en el que se desenvolverá. Algunos de estos factores que se subestiman y, en ocasiones, no se toman en cuenta son (Dalal y Chhillar, 2012):

- el número de clientes del servicio o aplicación

- la participación de transacciones monetarias en el software

- el tipo de uso de la aplicación

- el impacto en la vida de la gente si la aplicación falla

Con estos puntos inferimos que tanto la gerencia como la arquitectura del software desempeñan un papel muy importante en el desarrollo de un proyecto de software exitoso, ya que se debe tener una visión global del sistema y del medio ambiente en el que prestará sus servicios. Como vemos, la concurrencia de usuarios en la aplicación es uno de los puntos que, por lo general, no se tienen en cuenta, pero que son de vital importancia, ya que, cuando el software sea puesto en el ambiente de producción, podrá ser consultado por tantos usuarios que la arquitectura no estará preparada para la cantidad de solicitudes en un momento dado y no podrá dar un servicio adecuado o incluso se podría «caer» el servicio por completo.

La interoperabilidad entre servicios que realizan transacciones monetarias puede ser menospreciada en ocasiones en el sentido de que puede no tomarse en cuenta la evaluación de calidad de estos servicios externos. La funcionalidad del software que se está desarrollando debe interactuar con otras aplicaciones que también realizan operaciones financieras y donde sus resultados sirven como insumos a los cálculos de la aplicación que se está implementando (SAP, 2012). Sin embargo, se debería evaluar qué tan confiables son los procesos y resultados de estas otras aplicaciones con las que se tiene este tipo de interoperabilidad. 
Si el software es financiero, parece obvia su funcionalidad: administrar servicios que tienen que ver con el dinero y sus movimientos. Sin embargo, el razonamiento debería ir más allá, es decir, ya que la aplicación maneja dinero, se debería tener en cuenta la exactitud de los cálculos (Haselkorn, 2013), la velocidad de respuesta de la aplicación, la seguridad tanto del software como del hardware involucrado, etc.

Se piensa en todas las características que debería tener el software para cumplir con las necesidades de la gente. Sin embargo, se debe hacer un énfasis importante en las consecuencias que puede tener un fallo de la aplicación en la vida de los clientes. Si se trata de una aplicación financiera, un error puede afectar las condiciones financieras de un cliente en un momento dado, lo que puede derivar en su capacidad de compra, en la falta de pago de servicios públicos que garantizan su calidad de vida personal, en el préstamo de valores vitales como su servicio de salud y el de su familia, puede afectar el estado de compras o ventas de las empresas que utilicen el servicio, puede repercutir en la liquidación de nómina de una empresa, puede influir sobre las negociaciones entre compañías al no poder realizar transferencias o al realizar cálculos erróneos (King, 2014), etc. Este puede llegar a ser un gran listado de consecuencias de un fallo en el sistema financiero, el cual debería siempre ser tomado en cuenta en el desarrollo de un producto software que maneje dinero.

Dentro de los factores de éxito en los proyectos de software financiero que se han estudiado y que también son aplicables a la mayoría del software en general, se pueden enumerar estos, los cuales pueden ser los más relevantes (Project Smart, 2014):

- involucrar al usuario

- apoyo a la gestión ejecutiva

- claridad en la especificación de requerimientos

- adecuada planeación 
- expectativas realistas

- manejo de hitos de proyecto más pequeños

Involucrar al usuario en el desarrollo de software financiero podría no parecer una opción viable por cuestiones de seguridad. Sin embargo, la definición de usuario en este caso es mucho más amplia: el usuario interno es alguien que conoce de los procesos financieros, cómo realizarlos y para qué realizarlos. Estos usuarios internos, conocedores de temas financieros, son esenciales durante el proceso entero del desarrollo del software, de este modo es imprescindibles en la etapa de análisis de requerimientos y en el proceso de revisión de calidad y aprobación del proyecto, ya que estos son los que conocen del negocio. Los clientes y el equipo de desarrollo deberían trabajar juntos durante el proyecto (Beck, 2001).

El apoyo a la gestión ejecutiva es importante, puesto que la gerencia debe guiar todo el proceso de desarrollo para observar adecuadamente el entorno y tomar acciones y que estas sean tomadas en cuenta y acatadas por todos los involucrados para obtener una visibilidad completa del proyecto.

La claridad en la especificación de requerimientos es esencial para cumplir con la funcionalidad que el cliente necesita. Se debe hacer énfasis en la «necesidad» real, no en lo que el interesado quiere, ya que en un momento dado puede que él quiera algo que en realidad no le sirve y no va a solucionar el problema que tiene, se puede decir que «la gente no sabe lo que quiere hasta que se les muestra» (Jobs, 1998). Estos requerimientos serán los criterios de aceptación para dar el aval al inicio, la ejecución y la puesta en producción del software, es decir, son su razón de ser. 
Una adecuada planeación va de la mano con el hecho de tener unas expectativas realistas, se debe tener en cuenta que para que el proyecto culmine con éxito ha de tener fijadas unas metas claras, alcanzables y logrables en el tiempo estimado. Muchas veces, es útil manejar un gran proyecto en secciones más pequeñas, hitos del proyecto con unas entregas específicas que realizar hasta culminar el proyecto (Yang, He, Li, Wang y Boehm, 2008).

En el estudio realizado por Wiklund y Pucciarelli (2009), no solo se indican las razones principales de los problemas en un proyecto que llevan al fracaso de este, sino que se analizan desde la pregunta ¿por qué los proyectos tienen éxito? Y con este planteamiento se indica lo que se debe hacer para aumentar las posibilidades de éxito en un proyecto:

- Planificación y dirección de proyecto pobre: al ser la planificación y dirección del proyecto un factor clave para el éxito del proyecto, se requiere incluir procesos y herramientas de planificación y gestión de proyectos, teniendo en cuenta que una parte vital del proceso de planificación es asignar a las personas adecuadas a la tarea correcta, a quienes se dejarán claros los objetivos y las responsabilidades.

- Comunicación insuficiente: se debe contar con informes claros y objetivos, a fin de mantener comunicación constante con todos los interesados, generar participación activa; no se debe despreciar ninguna acción, todo cuenta, como tener agendas organizadas, preparar las reuniones y tener canales de comunicación definidos oficialmente.

- Gestión ineficaz: implementar un modelo de gestión de cambios ligero, pero que permita a la organización conocer el impacto y la evolución de las necesidades del proyecto, contar con herramientas para la evaluación de riesgos, además tener medidas de desempeño que deben estar incluidas en los informes de avance.

- No alineación entre la dirección del proyecto y los interesados: la construcción de confianza y claridad entre la dirección del proyecto y todos sus interesados es fundamental para 
un resultado exitoso. Se deben tener procesos que permitan tener enfocada la comunicación de las partes interesadas, y en etapas tempranas del proyecto, deben realizarse reuniones presenciales en las que se genere compromiso con este.

- Participación ineficaz de la alta gerencia: la participación de la alta gerencia no solo debe darse al inicio del proyecto; se ha de mantener comunicación constante e involucrar en procesos donde se deban tomar decisiones relevantes para el desarrollo del proyecto.

- Falta de habilidades sociales y capacidad de adaptación: se deben generar mecanismos de «padrinos» donde el personal especializado de la organización guía a personal nuevo. También dentro del calendario del proyecto se debe contar con capacitaciones y reclutar personal tanto interno como externo a la organización con conocimiento especializado para las necesidades del proyecto, entendiendo que no se logrará un buen resultado si no se cuenta con un equipo calificado.

- Deficiencia o falta de metodología y herramientas: los proyectos exitosos se basan en metodologías y herramientas de gestión, enfoque que suele aumentar la precisión y permitir la automatización de tareas.

\section{Hipótesis}

I $\mathrm{n}$ la presente investigación, se plantean las siguientes hipótesis:

- La falta de análisis de las consecuencias de errores en los proyectos podría llevar a perjudicar a los clientes y a la organización. El hecho de tomar como referencia las experiencias ocurridas en proyectos similares reduciría la probabilidad de fallar en la implementación de presentes y futuros proyectos.

- El hecho de no involucrar al usuario en el proceso podría hacer que los proyectos no sean exitosos. 
- Implementar todo lo que el cliente «quiere» podría no ir acorde con lo que el cliente «necesita».

- Al tener en cuenta los factores críticos de éxito y fracaso en el ciclo de vida del proyecto, se podría mejorar en realidad la probabilidad de éxito.

- No incluir metodologías o herramientas que brinden soporte a la gestión a lo largo del proyecto podría reducir las probabilidades de éxito este.

- La gestión de las comunicaciones por parte del gerente del proyecto podría ser un factor relevante para la salud y para el éxito del proyecto.

\section{Propuesta}

Dasándose en la información recolectada en la investigación sobre Dlos factores encontrados en el análisis realizado, a continuación se proponen algunas acciones como métodos y procedimientos para fortalecer los factores de éxito en los proyectos de software en Colombia.

Uno de los factores de fracaso, y que se vive a diario en los proyectos de software en el país, es el hecho de que las empresas desean siempre «funcionalidad» y este «objetivo» prima sobre todas las demás características por tener en cuenta durante el inicio, el transcurso y la finalización del proyecto, incluso sobre la «calidad». Es importante destacar que la calidad toma tiempo y debe ser una característica primordial de cualquier tipo de software, ya que de este depende la operación de las empresas al manejar sus procesos de negocio.

Los clientes presionan para que su software se termine lo más pronto posible, ya que tienen la falsa creencia de que esto los va a hacer más competitivos. La labor del gerente en este aspecto es crear conciencia en el cliente y hacerle ver que, si el software se hace rápido y en los tiempos que él estipula, se pueden cometer errores graves que afecten la calidad del 
producto, lo que hará que el software no cumpla con los requerimientos planteados de forma idónea y esto puede traer consecuencias en su negocio donde se puede ver afectada la operación diaria, las finanzas o incluso la credibilidad ante los clientes externos. La moraleja en esta reflexión es la siguiente: la calidad toma tiempo, pero el tiempo invertido en esta tarea proporcionará las mayores ventajas para la organización, y esto es lo que en realidad la hará más competitiva.

Aunque en Colombia hay fábricas de software que intentan aplicar de la mejor forma posible las metodologías de desarrollo de software tradicional como el método en cascada y sus derivados, se observa que no son muy eficientes debido la sobrecarga de trabajo en procesos que no aportan valor al proyecto como tal, por ejemplo el exceso en el manejo de la documentación. No se pretende demostrar que la administración de la documentación es inútil, ya que esta es un pilar sobre el que se cimenta la gestión y transferencia de conocimiento, sino que lo que se intenta decir es que el hecho de gastar demasiado tiempo y energía en crear y mantener documentos nos hace menos eficientes, además de reducir el interés que pueden tener los miembros de los equipos de proyectos analistas funcionales, desarrollo, calidad y todas la áreas relacionadasen consultar esta información por ser densa y de difícil consulta. Los gerentes deberían ser conscientes de tener la documentación necesaria para cada componente y fase del proyecto, de este modo esta es lo más clara y concisa posible.

El apego excesivo que se puede tener con las metodologías tradicionales puede hacer que se pierdan oportunidades de mejora en los procesos involucrados en el proyecto. Las metodologías ágiles contienen una gran cantidad de sugerencias y prácticas de mejora en estos procesos, como el fortalecimiento de la comunicación entre los miembros del equipo de proyecto por medio de la comunicación directa entre las personas discutiendo cara a cara, la sincronización del equipo diariamente para detectar problemas que deben ser solucionados con prioridad y para facilitar el seguimiento del avance del proyecto, la involucración del 
usuario durante todo el proceso de desarrollo, la división de un proyecto en varias fases cuyos objetivos son realizables - divide y vencerásy la obtención de feedback del cliente por medio de entregas parciales que muestran adelantos y aseguran victorias tempranas. La adecuada implementación de estas prácticas puede ayudar a mejorar drásticamente el estado de los proyectos, ya que se intentan contrarrestar directamente varios factores de fracaso comunes en los proyectos de software.

Un empleado feliz es un empleado productivo. Se debe realizar un análisis tanto de la cultura empresarial como de cada individuo para determinar qué puede hacer que los miembros del equipo se sientan cómodos con su trabajo y felices de realizarlo. Es importante aplicar

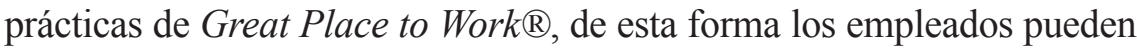
llegar a ser más eficientes, no se van a sentir intimidados o presionados, lo que favorecerá su creatividad, productividad e, incluso, su honestidad. A pesar de esto, se debe ser consciente de que cada persona es diferente y que hay gente que tiende a abusar de las comodidades dadas por el empleador. Es necesario detectar a estos individuos y hacerles caer en cuenta de que las facilidades proporcionadas para ellos tienen como objetivo hacer que mejoren en su trabajo y por este motivo deben dar resultados a la empresa. Esto hace parte del cambio productivo y evolutivo en la cultura empresarial.

Otro factor clave que influencia el éxito o fracaso de los proyectos de software en Colombia es el conocimiento que reside en los miembros del equipo de proyecto. La carencia de conocimientos de algún miembro del equipo en algún área vital para la ejecución del proyecto es un punto que debe ser de gran interés para una adecuada gestión del proyecto, ya que esto puede derivar en malas interpretaciones de los requerimientos del usuario, fallas de diseño y comunicación, subestimación de las tareas por desarrollar en el proyecto, mal diseño de pruebas, etc. Muchas veces la prisa que se tiene para terminar los proyectos hace que los involucrados tengan que aprender sobre temas puntuales de tecnología sobre la marcha, 
pero este aprendizaje puede no estar guiado y se puede incurrir en malas prácticas dentro de la propia tecnología o framework.

Se debe velar por que los miembros del equipo tengan las suficientes herramientas de conocimiento como para realizar adecuadamente su trabajo. Esto es muy importante en los gerentes, puesto que estos por lo general, aunque tienen un buen manejo de tecnología blanda para gestionar el proyecto, pueden llegar a menospreciar los conocimientos en tecnología dura. Un gerente que desee tener la verdadera visión global del proyecto y planear el esfuerzo adecuadamente debe tener conocimientos en ambos tipos de tecnología y estos deben ser lo bastante fuertes como para producir software de la más alta calidad y en los tiempos y costos convenidos.

De acuerdo con lo analizado en la documentación técnica, se logra identificar que los factores de éxito y de fracaso en los proyectos están relacionados con determinadas fases del ciclo de vida del proyecto (Tabla 1). 
Tabla 1. Factores de éxito y su relación con determinadas fases del ciclo de vida del proyecto

\begin{tabular}{|c|c|c|c|c|c|}
\hline Factor/fase & Iniciación & Planeación & Ejecución & Control & Cierre \\
\hline \multirow{4}{*}{ De éxito } & $\begin{array}{l}\text { Realizar una ade- } \\
\text { cuada evaluación y } \\
\text { selección de pro- } \\
\text { veedores basándo- } \\
\text { se en la experiencia } \\
\text { y calidad ofrecida } \\
\text { por cada uno. }\end{array}$ & $\begin{array}{l}\text { Definir un plan de } \\
\text { control de cam- } \\
\text { bios al alcance } \\
\text { en acuerdo con el } \\
\text { usuario. }\end{array}$ & $\begin{array}{l}\text { Realizar entregas } \\
\text { tempranas al } \\
\text { usuario para vali- } \\
\text { dar la satisfacción } \\
\text { y cumplimiento de } \\
\text { los requisitos. }\end{array}$ & $\begin{array}{l}\text { Identificar y } \\
\text { monitorear los } \\
\text { riesgos de ma- } \\
\text { nera oportuna. }\end{array}$ & $\begin{array}{l}\text { Generar lecciones } \\
\text { aprendidas tanto de } \\
\text { aspectos positivos } \\
\text { como de aspectos } \\
\text { negativos sobre } \\
\text { el desarrollo del } \\
\text { proyecto. }\end{array}$ \\
\hline & $\begin{array}{l}\text { Asignar un presu- } \\
\text { puesto acorde con } \\
\text { el tamaño del pro- } \\
\text { yecto sin sacrificar } \\
\text { calidad por costo. }\end{array}$ & $\begin{array}{l}\text { Asignar recursos } \\
\text { con conocimientos } \\
\text { en el negocio y en } \\
\text { las tecnologías por } \\
\text { utilizar en el desa- } \\
\text { rrollo del proyecto. }\end{array}$ & $\begin{array}{l}\text { Mantener moti- } \\
\text { vado el personal } \\
\text { que labora en el } \\
\text { proyecto. }\end{array}$ & $\begin{array}{l}\text { Realizar segui- } \\
\text { miento al plan } \\
\text { de control de } \\
\text { calidad y validar } \\
\text { con el usuario la } \\
\text { aceptación del } \\
\text { producto. }\end{array}$ & \\
\hline & $\begin{array}{l}\text { Involucrar al usuario } \\
\text { desde la etapa de } \\
\text { inicio del proyecto. }\end{array}$ & $\begin{array}{l}\text { Definir la tecnolo- } \\
\text { gía por utilizar de } \\
\text { manera adecuada, } \\
\text { teniendo en cuenta } \\
\text { las necesidades } \\
\text { del negocio y evi- } \\
\text { tando cambios de } \\
\text { esta en el desarro- } \\
\text { llo del proyecto. }\end{array}$ & $\begin{array}{l}\text { Evitar que los } \\
\text { mismos recursos } \\
\text { laboren jornadas } \\
\text { extenuantes sin } \\
\text { reconocimiento } \\
\text { alguno. }\end{array}$ & & $\begin{array}{l}\text { Realizar una buena } \\
\text { gestión del cambio } \\
\text { dentro de la orga- } \\
\text { nización en busca } \\
\text { de la aceptación del } \\
\text { nuevo software. }\end{array}$ \\
\hline & & $\begin{array}{l}\text { Involucrar al } \\
\text { usuario de manera } \\
\text { activa en el pro- } \\
\text { yecto, de manera } \\
\text { que se refleje su } \\
\text { participación en las } \\
\text { actividades descri- } \\
\text { tas en el plan de } \\
\text { trabajo planteado. }\end{array}$ & $\begin{array}{l}\text { Incluir al usuario } \\
\text { en las pruebas } \\
\text { del sistema para } \\
\text { obtener su acep- } \\
\text { tación. }\end{array}$ & & \\
\hline
\end{tabular}


Tabla 1. Factores de éxito y su relación con determinadas fases del ciclo de vida del proyecto (continuación)

\begin{tabular}{|c|c|c|c|c|c|}
\hline Factor/fase & Iniciación & Planeación & Ejecución & Control & Cierre \\
\hline \multirow{3}{*}{ De fracaso } & $\begin{array}{l}\text { Alcance mal defi- } \\
\text { nido debido a no } \\
\text { involucrar al usuario } \\
\text { en esta etapa. }\end{array}$ & $\begin{array}{l}\text { Aceptar las fechas } \\
\text { de finalización del } \\
\text { proyecto propues- } \\
\text { tas por el usuario } \\
\text { sin antes realizar la } \\
\text { debida estimación. }\end{array}$ & $\begin{array}{l}\text { No reconocer el } \\
\text { esfuerzo adicional } \\
\text { de los participan- } \\
\text { tes en el proyecto. }\end{array}$ & $\begin{array}{l}\text { No gestionar de } \\
\text { manera correcta } \\
\text { los riesgos del } \\
\text { proyecto. }\end{array}$ & \multirow{3}{*}{$\begin{array}{l}\text { En algunas orga- } \\
\text { nizaciones, debido } \\
\text { al clima organiza- } \\
\text { cional, los gerentes } \\
\text { de proyecto no } \\
\text { aceptan que fraca- } \\
\text { saron por miedo a } \\
\text { ser retirados de la } \\
\text { organización. }\end{array}$} \\
\hline & $\begin{array}{l}\text { Asignar un presu- } \\
\text { puesto inadecuado } \\
\text { para el proyecto en } \\
\text { busca de la econo- } \\
\text { mía desestimando } \\
\text { la calidad. }\end{array}$ & $\begin{array}{l}\text { No definir un plan } \\
\text { de gestión para } \\
\text { los controles de } \\
\text { cambios. }\end{array}$ & $\begin{array}{l}\text { No tener en } \\
\text { cuenta las alertas } \\
\text { generadas por los } \\
\text { participantes del } \\
\text { proyecto. }\end{array}$ & $\begin{array}{l}\text { Omitir las leccio- } \\
\text { nes aprendidas } \\
\text { de proyectos } \\
\text { similares. }\end{array}$ & \\
\hline & & $\begin{array}{l}\text { Definir una me- } \\
\text { todología que no } \\
\text { se ajusta en el } \\
\text { proyecto. }\end{array}$ & $\begin{array}{l}\text { Inclusión de } \\
\text { personal sin } \\
\text { conocimiento en } \\
\text { el proyecto en las } \\
\text { etapas críticas en } \\
\text { busca de cum- } \\
\text { plir con la fecha } \\
\text { comprometida de } \\
\text { entrega. }\end{array}$ & & \\
\hline
\end{tabular}

Fuente. Elaboración propia. 
Se realizaron entrevistas - anexo 1-a un grupo de gerentes de proyectos, directores y coordinadores de las áreas de tecnología de cuatro bancos que tienen presencia en Colombia, tras lo cual se encontraron los siguientes factores:

A. Es «normal» que los proyectos terminen después del tiempo inicialmente planeado; por la cultura del país, la preocupación está más enfocada en que el producto o servicio que genere el proyecto funcione.

B. Se identificaron los siguientes factores de fracaso en los proyectos:
a. Alcance mal definido.
b. No involucrar a todos los interesados.
c. Falta de conocimiento y experiencia del equipo del proyecto.
d. Falta de apoyo de la alta gerencia.
e. Ausencia de un proceso de control de cambios.
f. Cambios en la tecnología.
g. Tiempos de respuesta impuestos desde la alta gerencia.
h. Ausencia de metodología y herramientas de apoyo parta gestión.
i. Renuncia de recursos clave.
j. Mala identificación de riesgos.

C. Se identificaron los siguientes factores de éxito en los proyectos:
a. Alta dedicación en la fase de planeación.
b. Equipo de proyecto con conocimiento del negocio y técnico avanzado.
c. Definición precisa de alcance, requerimientos completos.
d. Gestión total de interesados, de tal manera que se involucren en todas las fases del proyecto.


e. Herramientas automatizadas para gestión y control del proyecto.

f. Definición del alcance del proyecto sincronizada con los objetivos de la organización, de este modo es apoyado por la alta gerencia.

g. Metodología de gestión de principio a fin del proyecto.

h. Comité de control de cambios.

i. Habilidades comunicativas del gerente de proyecto.

D. Se solicitó que de los últimos proyectos donde estuvieron indicaran si se había o no presentado retrasos en los tiempos de entrega. Se encontró que de 44 proyectos el $41 \%$ habían tenido un retraso de más de un año y solo el $7 \%$ habían cumplido el plazo inicialmente definido (Figura 6).

Figura 6. Desfase en tiempo de proyectos en los que estuvieron involucrados los encuestados

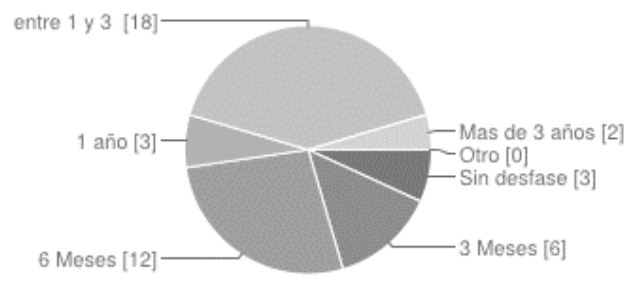

$\begin{array}{lrr}\text { Sin desfase } & 3 & 7 \% \\ 3 \text { Meses } & 6 & 14 \% \\ 6 \text { Meses } & 12 & 27 \% \\ \text { 1 año } & 3 & 7 \% \\ \text { entre 1 y 3 años } & 18 & 41 \% \\ \text { Mas de 3 años } & 2 & 5 \% \\ \text { Otro } & 0 & 0 \%\end{array}$

Fuente. Elaboración propia.

E. Se identificó que en la mayoría de los casos los gerentes al iniciar el proyecto no tenían experiencia en el tema y, en ocasiones, no contaban con el equipo adecuado para finaliza el proyecto de la forma esperada. 
F. Dentro de los retos más relevantes identificados se tienen:

a. Cumplir con la triple restricción, entregar todo lo comprometido, en el tiempo planeado, dentro del presupuesto definido y con la calidad esperada.

b. Tener buena documentación de requerimientos.

c. Contar con un equipo idóneo, con todos los conocimientos necesarios.

d. Generar compromiso de todas las partes interesadas.

La tabla 2 muestra los factores de éxito y de fracaso que se tienen en común entre el conocimiento técnico-académico y el empírico vivido por gerentes en la banca colombiana. 
Tabla 2. Factores de éxito y fracaso comunes entre el conocimiento técnico-académico y empírico

\begin{tabular}{|c|c|c|}
\hline Factor & Conocimiento técnico & Conocimiento empírico \\
\hline \multirow{10}{*}{ De éxito } & $\begin{array}{l}\text { Centrar toda la atención en la gestión } \\
\text { de los interesados y en la estrategia } \\
\text { más que en el presupuesto y el cro- } \\
\text { nograma. }\end{array}$ & $\begin{array}{l}\text { Gestión total de interesados, de tal } \\
\text { manera que se les involucre en todas } \\
\text { las fases del proyecto. }\end{array}$ \\
\hline & $\begin{array}{l}\text { Dominio de la tecnología utilizada en el } \\
\text { proyecto para asegurar recursos tanto } \\
\text { internos como externos. }\end{array}$ & $\begin{array}{l}\text { Equipo de proyecto con conocimiento } \\
\text { del negocio y técnico avanzado. }\end{array}$ \\
\hline & $\begin{array}{l}\text { Creación de equipos eficaces, con } \\
\text { motivaciones alineadas a los objetivos } \\
\text { del proyecto. }\end{array}$ & \\
\hline & $\begin{array}{l}\text { Buenas prácticas de gestión de pro- } \\
\text { yectos como ciclos de entrega cortos y } \\
\text { rigurosos controles de calidad. }\end{array}$ & $\begin{array}{l}\text { Metodología de gestión de principio a } \\
\text { fin del proyecto. }\end{array}$ \\
\hline & & $\begin{array}{l}\text { Herramientas automatizadas para } \\
\text { gestión y control del proyecto. }\end{array}$ \\
\hline & & $\begin{array}{l}\text { Definición del alcance del proyecto } \\
\text { sincronizada con los objetivos de la } \\
\text { organización, de este modo es apoyado } \\
\text { por la alta gerencia. }\end{array}$ \\
\hline & & $\begin{array}{l}\text { Definición precisa de alcance, requeri- } \\
\text { mientos completos. }\end{array}$ \\
\hline & & $\begin{array}{l}\text { Alta dedicación en la fase de planea- } \\
\text { ción. }\end{array}$ \\
\hline & & Comité de control de cambios. \\
\hline & & $\begin{array}{l}\text { Habilidades comunicativas del gerente } \\
\text { de proyecto. }\end{array}$ \\
\hline
\end{tabular}


Tabla 2. Factores de éxito y fracaso comunes entre el conocimiento técnico-académico y empírico (continuación)

\begin{tabular}{|c|c|c|}
\hline Factor & Conocimiento técnico & Conocimiento empírico \\
\hline \multirow{15}{*}{ De fracaso } & $\begin{array}{l}\text { Los gerentes del proyecto no entien- } \\
\text { den las necesidades de los usuarios. }\end{array}$ & \\
\hline & $\begin{array}{l}\text { No está bien definido el alcance del } \\
\text { proyecto. }\end{array}$ & Alcance mal definido. \\
\hline & $\begin{array}{l}\text { No existe un proceso formal de control } \\
\text { de cambios y por ende estos se ges- } \\
\text { tionan mal. }\end{array}$ & $\begin{array}{l}\text { Ausencia de un proceso de control de } \\
\text { cambios. }\end{array}$ \\
\hline & $\begin{array}{l}\text { Se realizan cambios en las tecnolo- } \\
\text { gías inicialmente definidas. }\end{array}$ & Cambios en la tecnología. \\
\hline & Se presentan cambios en el negocio. & \\
\hline & $\begin{array}{l}\text { Definición de tiempos y cronogramas } \\
\text { no realistas. }\end{array}$ & \\
\hline & Usuarios resistentes. & \\
\hline & $\begin{array}{l}\text { Pérdida de respaldo del patrocinador } \\
\text { del proyecto. }\end{array}$ & \\
\hline & $\begin{array}{l}\text { El proyecto no cuenta con el equipo } \\
\text { de trabajo que tenga las habilidades } \\
\text { necesarias para este. }\end{array}$ & $\begin{array}{l}\text { Falta de conocimiento y experiencia del } \\
\text { equipo del proyecto. }\end{array}$ \\
\hline & $\begin{array}{l}\text { El gerente ignora las buenas prácticas } \\
\text { y lecciones aprendidas. }\end{array}$ & $\begin{array}{l}\text { Ausencia de metodología y herramien- } \\
\text { tas de apoyo para gestión. }\end{array}$ \\
\hline & & $\begin{array}{l}\text { Tiempos de respuesta impuestos desde } \\
\text { la alta gerencia. }\end{array}$ \\
\hline & & No involucrar a todos los interesados. \\
\hline & & Falta de apoyo de la alta gerencia. \\
\hline & & Renuncia de recursos clave. \\
\hline & & Mala identificación de riesgos. \\
\hline
\end{tabular}

Fuente. Elaboración propia. 


\section{Conclusiones}

Tos resultados permiten validar las hipótesis planteadas al inicio de la Uinvestigación. De este modo, se encuentran los siguientes elementos:

La primera hipótesis planteada abordaba las consecuencias de no llevar históricos de los proyectos y de las lecciones aprendidas de tal forma que se tuviera una fuente para realizar análisis. Se logra concluir que las organizaciones que no tienen procesos que permitan documentar los eventos tantos positivos como negativos en los proyectos ejecutados tienden a repetir los mismos errores o a implementar soluciones más costosas.

La segunda hipótesis indicaba que el no involucrar al usuario final en el desarrollo del proyecto podría llevar al fracaso de este. Dentro de los factores de fracaso identificados esta la lejanía del proyecto con el usuario final, donde se levantan los requerimientos y se vuelve a tener comunicación hasta la etapa en que se entrega el producto o servicio. Es importante tener una relación y comunicación constante con el usuario de tal forma que él pueda ver avance del proyecto y entregue retroalimentación temprana al equipo del proyecto para mantener alineados sus objetivos. Sin embargo, esto debe estar enmarcado dentro de un proceso que permita garantizar que no se incluyan cambios en los requerimientos.

La tercera hipótesis plantea que no todo lo que el cliente desea es lo que necesita. Esto permite ver la responsabilidad del equipo del proyecto en lograr plasmar las necesidades del cliente en requerimientos que sí brinden la cobertura a esta necesidad con la calidad y la funcionalidad que apoye los procesos de cliente. Los proyectos en los que no se tiene un acercamiento y un buen análisis de requerimientos terminan por entregar un producto o servicio que no satisface las necesidades del cliente y que en algunas ocasiones no es aceptado ni usado. 
La cuarta hipótesis sugiere que, al tener en cuenta los factores de éxito y de fracaso en el ciclo de vida de los proyectos, puede aumentar las probabilidades de éxito. Con el análisis de la bibliografía técnica y teniendo en cuenta el conocimiento empírico de los gerentes entrevistados, se concluye que desa-rrollar un proyecto de software en el sector financiero implica un amplio conocimiento y desarrollo de habilidades que permitan tener bajo control las variables que se presentan en el proyecto. Sin embargo, es recomendable que durante las etapas iniciales del proyecto se tengan en cuenta los factores de éxito en los proyectos para enfocar allí los esfuerzos y de igual forma los factores de fracaso para definir estrategias y planes de trabajo que eliminen estos elementos y que permitan tener un control constante sobre ellos. Al planear un proyecto teniendo en cuenta estos factores, se logra tener un panorama más amplio de los obstáculos y las oportunidades que el proyecto debe manejar, y si desde su concepción se tienen en cuenta, se aumentarán las probabilidades de éxito.

La quinta hipótesis menciona la necesidad de tener metodo-logías o herramientas que apoyen los procesos propios del proyecto. Como resultado del análisis, se evidencia que los proyectos con metodologías son más organizados, permiten agilidad en las actividades y todo el equipo del proyecto conoce en detalle sus responsabilidades, además aquellos que cuentan con herramientas de gestión logran identificar de forma temprana y fácil problemas; antes de que tengan un mayor impacto, estas herramientas se convierten en algunos casos en elementos de control o generación de alertas a los diferentes interesados del proyecto.

La sexta hipótesis menciona la importancia de una buena gestión de comunicaciones. El gerente del proyecto tiene la responsabilidad de garantizar un flujo constante de toda la información teniendo en cuenta procesos como planeación, recopilación, creación, distribución y almacenamiento, donde el gerente invierte la mayor parte de su tiempo comunicándose con miembros del equipo, clientes, patrocinador, la alta gerencia, interesados internos y externos de la organización, de tal forma 
que la comunicación eficaz del gerente se convierte en un puente entre todos los interesados que pueden tener diferencias en experiencia, cultura, perspectivas del proyecto, influencia, posición en la organización, con los que pueden impactar o influenciar el proyecto o el resultado de este.

El análisis de los resultados permite concluir que existen factores de éxito en todas las etapas de un proyecto, los que deben verse dentro de la organización como conocimiento adquirido y que deben transformarse en base de conocimiento para la definición, planeación y desarrollo de cualquier proyecto. Es fundamental que los gerentes de proyecto conozcan esta información y tengan las habilidades y los conocimientos necesarios para sacar el mayor provecho de ellos.

Con respecto a los factores de fracaso, es importante llevar un registro de lecciones aprendidas donde se detalle lo que llevó al problema y que permita a nivel organizacional definir estrategias que mitiguen estas fallas. En algunos casos, estos elementos repercuten en mejoras a los procesos de la organización en la definición de proyectos, en nuevas bases de roles y tipos de equipos de proyecto necesarios para sortear los obstáculos que se presentan en ellos. Estos factores también permiten sensibilizar a la alta gerencia de tal forma que se redireccione la idea de que un proyecto de software es una inversión que requiere especial cuidado, relevancia y apoyo. Dentro de los factores de fracaso, se puede concluir que todos convergen en la definición de «la triple restricción», debido a que cualquier impacto o problema en general afecta el tiempo, alcance o costo y, en algunos casos, a los tres, lo que debe llevar al gerente del proyecto a definir elementos dentro de este que le permitan tener un control cercano y detallado de estos elementos para poder tomar decisiones con buenas bases de información, en busca de llevar el proyecto al éxito dentro de las variables esperadas que son entregar el producto o servicio en el tiempo acordado, con toda la funcionalidad o requerimientos definidos y dentro del presupuesto asignado. 
Los factores de éxito y de fracaso en proyectos del sector financiero colombiano que más se repiten son:

- La mayor parte de los factores de fracaso encontrados se presentan por un mal manejo y descuido de la triple restricción: alcance, tiempo y costo, por parte del gerente de proyecto.

- La falta de experiencia del gerente y del equipo de proyecto en el manejo de riesgos hace que los proyectos fracasen a mediano plazo.

- El dinamismo, la velocidad del mercado financiero y la exigencia de la alta gerencia hace que los proyectos no logren ejecutar todo el proceso de calidad para llevar a cabo la consecución del producto o servicio que se espera construir lo que causa falencias en su resultado final.

- El proyecto no está alineado con los objetivos misionales de la organización y por ende no logra obtener el apoyo ni los recursos económicos para llevarse a cabo.

- Por el estilo cultural del país, en algunos casos, se dejan elementos importantes de lado solo por el hecho de cumplir con la entrega de una funcionalidad acordada, con lo que se sacrifican atributos de calidad, relación con el cliente $\mathrm{y}$, en algunos casos, completitud, lo que ahonda en mayores costos.

Dentro de las mayores dificultades de la investigación, está la falta o poca bibliografía que existe sobre el desarrollo y las características de proyectos en el país, además los gerentes que se encuentran involucrados en proyectos de software del sector financiero no cuentan con el tiempo para desarrollar a fondo el análisis y la identificación de los factores. También por el tipo de sector se tiene cierto recelo en cuanto a confidencialidad y conocimiento adquirido para el manejo de sus proyectos. 
A continuación, se plantean algunas preguntas que se podrían desarrollar en el futuro en una investigación complementaria:

- ¿Cómo impulsar la estandarización y el manejo de proyectos en todo el país por sectores económicos?

- ¿Cómo generar cambio en la cultura de proyectos del país pasando de la gestión tradicional a una gerencia de proyectos moderna?

- ¿Qué estrategias se podrían implementar para generar mayor apoyo de la alta gerencia por los proyectos de software?

- ¿Cómo generar espacios de conocimiento para compartir experiencias en proyectos del sector financiero?

- ¿Cómo crear espacios de colaboración para el desarrollo de proyectos de software en el sector financiero? 


\section{Referencias}

Bahamón Lozano, J. H. (2003). Construcción de indicadores de gestión bajo el enfoque de sistemas. Sistemas \& Telemática, 1(1), 77, 87. Recuperado de https://doi.org/10.18046/syt.v1i1.1080

Bloch, M., Blumberg, S. y Laartz, J. (2012). Delivering large-scale IT projects on time, on budget, and on value. McKinsey on Business Technology, 27. Recuperado de https://bit.ly/3006B1O

Cano, W. G. (2006). Introducción a la gerencia de proyectos. Medellín: Instituto para el servicio. Recuperado de https://bit.ly/2Ji2GY4

Cortés Cortés, M. y Iglesias León, M. (2005). Generalidades sobre metodología de la investigación. Ciudad del Carmen, México: Universidad Autónoma del Carmen. Recuperado de https://bit.ly/2qknK85

Dalal, S. y Chhillar, R. S. (2012). Case studies of most common and severe types of software system failure. International Journal of Advanced Research in Computer Science and Software Engineering, 2(8), 341-347. Recuperado de https://bit.ly/2PO93Ug

Domínguez Fernández, B. (2007, abril 20). Fundamentos de gestión de proyectos efectiva. Recuperado de https://bit.ly/2PO99v6

Dorsey, P. (2005). Top 10 reasons why systems projects fail. Recuperado de https://bit.ly/2Ws99Ub

Evans, J. y Ganegoda, A. (2012). Classification of risks and management implications. Risk Management Today. Recuperado de https://bit. ly/2Y7TI3N

Haselkorn, E. (2013, julio 17). Data accuracy an important consideration for financial institutions going mobile. Experian. Recuperado de https://bit. ly/2Vh0OWO

Iso25000.com (s. f.). ISO/IEC 25010. Recuperado de https://bit.ly/2H51Z5y

Kelion, L. (2013, febrero 1). Why banks are likely to face more software glitches in 2013. BBC. Recuperado de https://bbc.in/2Ws9NRB 
King, L. (2014a, junio 3). Oops! Bad Software Code Knocks Down US Manufacturing, Dow Jones AND S\&P 500. Forbes. Recuperado de https://bit.ly/2VNOwVd

King, L. (2014b, junio 7). Terrible state of financial software code plagues investors. Forbes. Recuperado de https://bit.ly/2LrRXx0

Lyytinen, K. y Hirschheim, R. (1988). Information systems failures: A survey and classification of the empirical literature. En Oxford surveys in information technology (pp. 257-309). Nueva York: Oxford University Press. Recuperado de https://bit.ly/2vDwfeL

McManus, J. y Wood-Harper, T. (2007). Understanding the sources of information systems project failure. Management Services. Recuperado de https://bit.ly/2H90ot3

Ocampo, A. (2013, septiembre 27). Entrevista a Alexis Ocampo, una voz autorizada en la ingeniería de software. Revista Sistemas. Recuperado de https://bit.ly/2H4j8bz

Partnov, N. y Estier, T. (2009). E-commerce applications evolution issues: The case of e-banking. Recuperado de https://bit.ly/2H3vWQI

Project Management Institute (2013). Guía de los fundamentos para la dirección de proyectos (Guía del PMBOK®) (5. ${ }^{\mathrm{a}}$ ed.). Project Management Institute.

Project Smart (2014). The Standish Group Report: CHAOS. Recuperado de https://bit.ly/1JFKOwF

Real Academia Española (2014). Diccionario de la lengua española (23. ${ }^{\mathrm{a}}$ ed.). Madrid: Espasa. Recuperado de https://bit.ly/1xc5qat

Reel, J. S. (1999). Critical success factors in software projects. IEEE Software, 3, 18-23. Recuperado de https://bit.ly/2H5S3pV

Rockart, J. F. (1979). Chief executives define their own data needs. Harvard Business Review, 57(2), 81-93. Recuperado de https://bit.ly/2s2qkxK 
Romero López, R., Noriega Morales, S. A., Escobar Toledo, C. E. y Ávila Delgado, V. I. (2009). Factores críticos de éxito: una estrategia de competitividad. CULCyT: Cultura Cientifica y Tecnológica, 31, 5-14. Recuperado de https://bit.ly/2GZNBaW

Salinas Duarte, A. E. (2007). Obstáculos en la gestión de proyectos en tecnologías de información y comunicación: TIC y posibles soluciones. Recuperado de https://bit.ly/2H4jIrg

SAP (2012, junio 17). Overview of the most important industry standards for the banking industry. Recuperado de https://bit.ly/2H3nUqZ

Villegas, G. C. (1997). Gestión por factores críticos de éxito. Revista EAFIT, 33(105), 9-33. Recuperado de https://bit.ly/2J5UieP

Wiklund, D. y Pucciarelli, J. C. (2009). Improving IT project outcomes by systematically managing and hedging risk.

Yang, Y., He, M., Li, M., Wang, Q. y Boehm, B. (2008). Phase distribution of software development effort. En Proceedings of the Second ACMIEEE international symposium on Empirical software engineering and measurement (pp. 61-69). Nueva York: ACM. Recuperado de https://bit. ly/2JsSFHR 


\section{Anexo 1. Encuesta a gerentes de proyectos de software financiero}

1. ¿En los proyectos de software que ha participado se ha cumplido a satisfacción el plan de trabajo y tiempos con el que se dio inicio al proyecto?

2. Enumere diez factores que han llevado al fracaso al proyecto de software.

3. Enumere diez factores que usted crea llevarían al éxito los proyectos de software.

4. ¿Qué rescata de los proyectos en los que ha participado?

5. ¿Cuánto ha sido el desfase en tiempo de los proyectos de software en los que ha participado?

6. ¿Cuándo inició a dirigir el proyecto tenía experiencia como gerente de proyectos de software?

7. ¿Ha participado en proyectos que lleguen a culminarse o que se terminan pero el usuario no los utiliza?

8. ¿Qué tan importante es para usted la participación del usuario en el proyecto?

9. ¿En los proyectos que ha dirigido involucra al usuario?

10. ¿Cuál es el reto más importante al dirigir un proyecto de software?

11. ¿Cuáles son los elementos más importantes por tener en cuenta en el momento de planear un proyecto de software?

12. ¿Cuáles son los elementos más importantes en el momento de realizar seguimiento y control a un proyecto de software?

13. ¿Cuáles son los elementos más importantes durante la ejecución de un proyecto de software? 